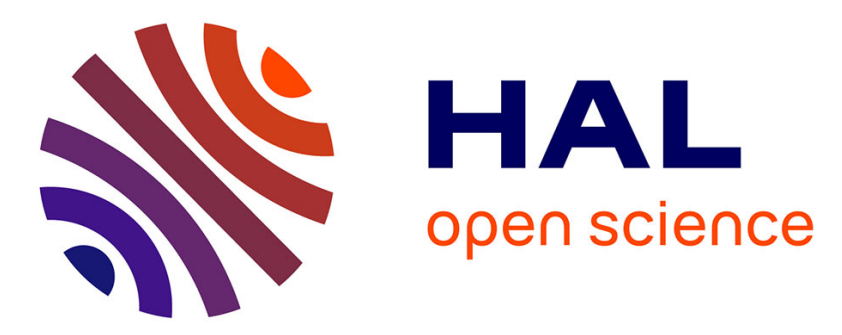

\title{
Comparison of predictions by mode II or mode III criteria on crack front twisting in three or four point bending experiments
}

\author{
Veronique Lazarus, F.-G. Buchholz, M. Fulland, J. Wiebesiek
}

\section{To cite this version:}

Veronique Lazarus, F.-G. Buchholz, M. Fulland, J. Wiebesiek. Comparison of predictions by mode II or mode III criteria on crack front twisting in three or four point bending experiments. International Journal of Fracture, 2008, 153 (2), pp.141-151. 10.1007/s10704-008-9307-2 . hal-01906229

\author{
HAL Id: hal-01906229 \\ https://hal.science/hal-01906229
}

Submitted on 26 Oct 2018

HAL is a multi-disciplinary open access archive for the deposit and dissemination of scientific research documents, whether they are published or not. The documents may come from teaching and research institutions in France or abroad, or from public or private research centers.
L'archive ouverte pluridisciplinaire $\mathbf{H A L}$, est destinée au dépôt et à la diffusion de documents scientifiques de niveau recherche, publiés ou non, émanant des établissements d'enseignement et de recherche français ou étrangers, des laboratoires publics ou privés. 


\title{
Comparison of predictions by mode II or mode III criteria on crack front twisting in three or four point bending experiments
}

\author{
V. Lazarus · F.-G. Buchholz · M. Fulland · J. Wiebesiek
}

Received: date / Accepted: date

\begin{abstract}
Whatever the external loading, a crack front in a solid tries to reach mode I loading conditions after propagation. In mode I+II, the crack kinks to annihilate mode II, kinking angle being well predicted by the principle of local symmetry (PLS) or by the maximum tangential stress criterion (MTS). In presence of mode III, the problem becomes three-dimensional and the proposed propagation criterion are not yet well proved and established. In particular in three point bending experiments (3PB) with an initially inclined crack, the crack twists around the direction of propagation to finally reach a situation of pure mode I. The aim of the paper is to compare the propagation paths predicted by two different criteria for 3PB fatigue experiments performed on PMMA. The first criterion developed by Schollmann et al (2002), is a three-dimensional extension of the MTS criterion and predicts the local angles that annihilates mode II and III at each point of the front. The second one developed by Lazarus et al (2001b), predicts an abrupt and then progressive twisting of the front to annihilate mode III. Due to presence of sign changing mode II and almost uniform mode III in the experiments, both criteria give good results. However, since mode III is predominant over mode II in the case under consideration, the global criterion gives better results. Nevertheless, the local type criterion seems to be of greater universality for practical engineering applications.
\end{abstract}

\section{Lazarus}

UPMC Univ Paris 6, Univ Paris-Sud, CNRS, UMR 7608, Lab FAST, Bat 502, Campus Univ, F-91405, Orsay, France

Tel.: +33-1-69158039

Fax: +33-1-69158060

E-mail: veronique.lazarus@upmc.fr

F.-G. Buchholz, M. Fulland, J. Wiebesiek Institute of Applied Mechanics, University of Paderborn, Pohlweg 47-49, D-33098 Paderborn, Germany E-mail: fus.buchholz@ tonline.de,fulland@fam.upb.de
Include keywords, PACS and mathematical subject classification numbers as needed.

Keywords Brittle fracture $\cdot$ mode II $\cdot$ mode III $\cdot$ Maximum tangential stress criterion $\cdot 3 \mathrm{D}$ crack path $\cdot 3 \mathrm{D}$ fracture criteria

\section{Introduction}

In homogeneous isotropic elastic media, except in some special conditions, it is well known that whatever the external loading, the crack front bifurcates in order to reach a situation of pure mode I as the crack propagates (Hull, 1999). A literature survey of mixed mode crack growth can be found in Oian and Fatemi (1996). Under mode (I+II) conditions, the crack kinks to annihilate mode II, as depicted in figure 5(c) The value of the corresponding kink angle can be obtained, for instance, by the Principle of local Symmetry (PLS) of Goldstein and Salganik (1974) or by the maximum tangential stress criterion (MTS) (Erdogan and Sih, 1963). In presence of mode III, to reach a situation of mode I, the crack front twists around the direction of propagation. The way this twisting occurs is case dependant : in some cases, it occurs gradually (fig. 1(a) and 1(c)], in some other cases abruptly (fig. 1(b) and 1(d); ; sometimes the whole front twists in one-piece (see fig. [1(a) and 1(b) and in some other cases, the front twists piecewise leading to a factory roof pattern (see fig. 1(c) and 1(d)] The conditions of transition between these several crack patterns remain an open question. For instance, gradually piecewise rotation has been observed by Sommer (1969), Hourlier and Pineau (1979); abruptly piecewise rotation by Palaniswamy and Knauss (1975). In Makabe et al (2006), depending on the loading conditions, both progressive and abrupt rotation have been observed. Abrupt piecewise twisting at a small length-scale 


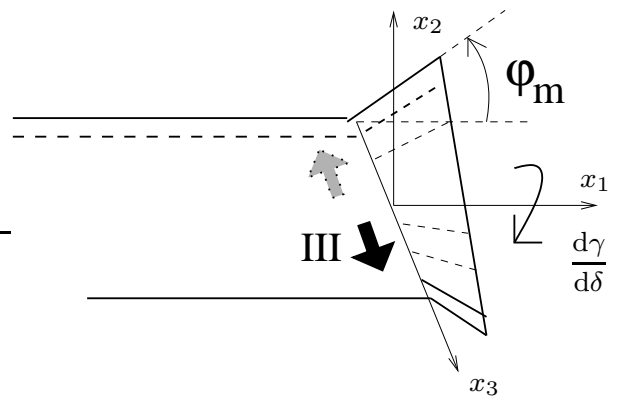

(a) Gradual one-piece twisting

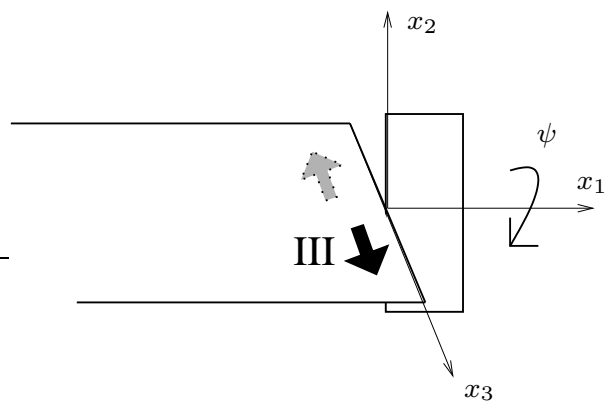

(b) Abrupt one-piece twisting

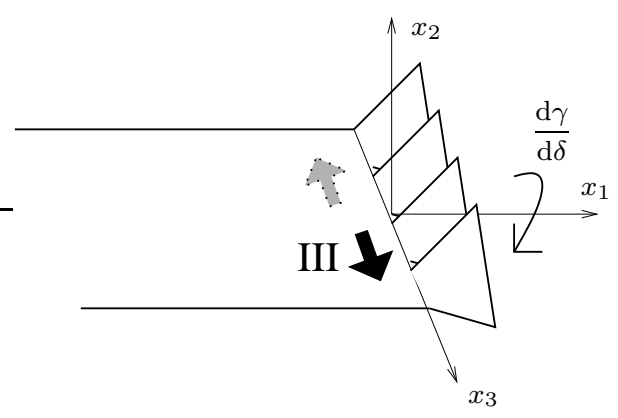

(c) Gradual piecewise twisting

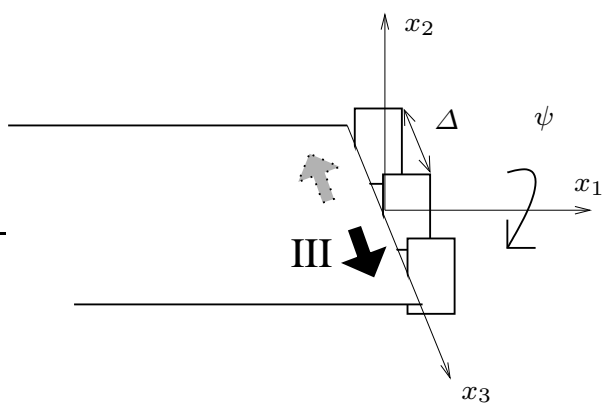

(d) Abrupt piecewise twisting

Fig. 1 Several basic propagation possibilities in presence of mode III

and progressive rotation of the whole crack front at a macroscopic length-scale, has been observed in three or four points bending (3PB or 4PB) experiments. Such experiments and observations have been made by Yates and Mohammed (1996) on steel specimens in fatigue, by Cooke and Pollard (1996), Buchholz et al. (1998; 2005)on PMMA blocks in fatigue, by
Lazarus and Leblond (1998), Lazarus et al (2001b) on PMMA specimens under monotonic increasing loading. These patterns can also be recognized on geological materials (Pollard et al, 1982; Avdin and Pollard, 1988) at different length-scales.

The qualitative way of crack propagation is well explained by its tendency to reach mode I conditions (Hull, 1993; Pook, 1995). But it is not sufficient to predict the propagation path quantitatively and a few fracture criteria have been proposed so far. Concerning the abrupt rotation of the crack front, several criteria have been proposed. But the only paper we are aware of, that compares the results with experimental findings is Cooke and Pollard (1996), who showed that all the criteria overestimate the real value. Lazarus et al (2001b) proposed the following fit:

$\psi=\frac{1}{4} \arctan \frac{2 K_{I I I}}{(1-2 \nu) K_{I}}$

by interpolation of their experimental data. In the special case of 3 or $4 \mathrm{~PB}$ experiments, the gradually rotation of the front, has been predicted, on the one hand, by Gravouil et al (2002) and Buchholz et al (2004) using MTS-criteria and by Lazarus et al. (1998, 2001b) using a criterion predicting the twisting rate of the whole crack front. Whereas the kink angles given by MTS criteria lead to the annihilation of mode II, the twisting rate of Lazarus et al. (1998, 2001b) leads to the annihilation of mode III. Hence it may be surprising that both criteria manage to find the experimental propagation path. Also the aim of this paper is to discriminate these two kind of criteria by comparing the predictions of the MTS criterion (Erdogan and Sih, 1963) or its 3D extension (Schollmann et al, 2002) and those of the criterion proposed by Lazarus et al. (1998; 2001b) to 3PB fatigue experiments performed on PMMA.

These experiments are presented in section 2 Both modes II and III are present in the experiments. By symmetry, mode II Stress Intensity Factor (SIF) takes opposite signs at each side of the front, hence MTS criterion (or equivalent 3D ones, see section 3.2 predicts an opposite sign of the kink angle. Mode III yields a uniform twisting of the whole front by Lazarus et al. criterion (section 3.3. Hence, at least qualitatively, the same kind of twisting propagation path is expected and the quantitative comparison (section 4 ) with the experiments shows that both criteria allow to recover quite well the experimental values. Nevertheless, two facts shall limit the extensive use of MTS criterion (or equivalent ones such as PSL or 3D-MTS generalisation) in presence of mode III although MTS criteria seem to be sufficient for any practical engineering purposes. The first fact is that increasing mode III over mode II rate, gives advantage to Lazarus et al. criterion toward the MTS one, and the second that MTS criterion predicts well the kink angle near the free surfaces but too low ones near center of the front. 


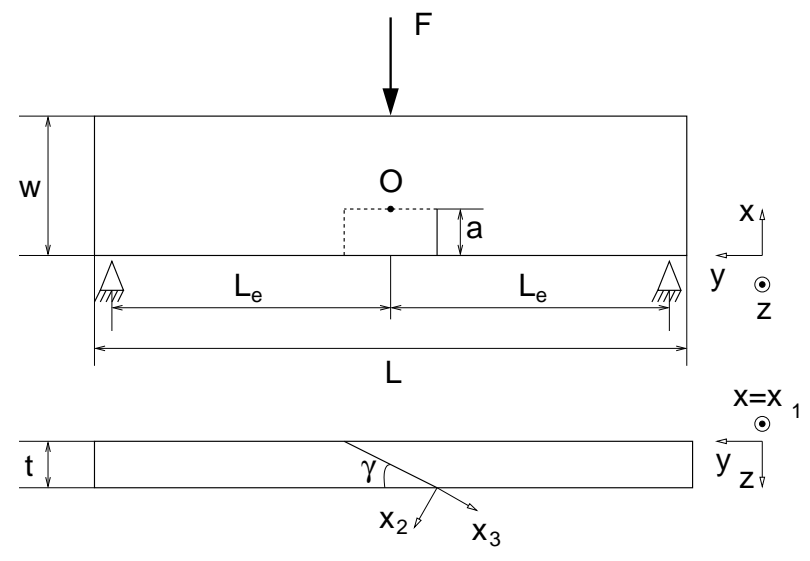

Fig. 2 3PB-specimen with an inclined initial notch

\section{Experiments}

The experiments are 3PB experiments performed on PMMA specimens containing an initial inclined slit (Fig. 22. The geometrical parameters of the 3PB-specimens are as follows: length $L=260 \mathrm{~mm}, 2 L_{e}=240 \mathrm{~mm}$, thickness $t=10 \mathrm{~mm}$, width $W=60 \mathrm{~mm}$, normalized crack length $a / w=1 / 3$, angles of the inclined planes of the initial cracks or notches with $\gamma=75^{0}, 60^{\circ}$ and $45^{\circ}$. For the simulation the material parameters are given by the experimental specimens from PMMA with Young modulus $E=2800 \mathrm{~N} / \mathrm{mm}^{2}$ and Poisson ratio $\nu=0.38$. The specimens are subject to a cyclic lateral force of $F=2.4 \mathrm{kN}$ and the stress ratio of the cyclic loading is $R=0.1$.

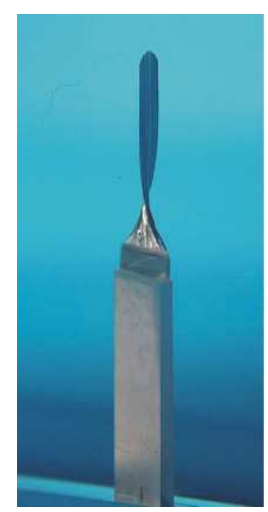

(a) Global view for (b) Final view for several angles $\gamma=45^{0}$

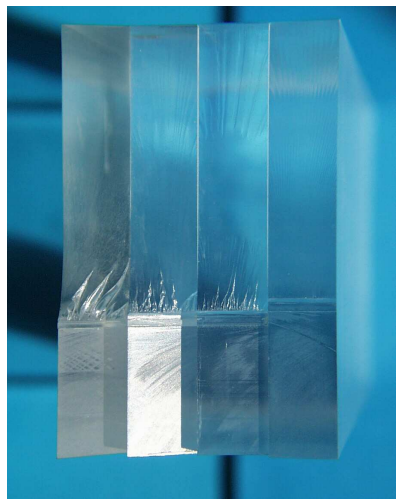

$\gamma=45^{0}, 60^{0}, 75^{0}, 90^{0}$
Fig. 3 Gradual one-piece crack twisting

For $\gamma=90^{\circ}$, the initial notch is located in the middle $(x, z)$ plane of the specimen. Due to symmetry reasons, the crack is then loaded in mode I only and crack propagates straight and breaks the specimen in two equivalent pieces. For $\gamma$ less than $90^{\circ}$ the initial notch is loaded in both tension and out-of-plane shear, and the crack bifurcates during propagation. Pictures of the propagation path (fig. 3) show that the crack front gradually twists around the direction of propagation to finally reach the middle mode I plane $(x, z)$ of the specimen. Hence, crack propagates to annihilate shear loading. One can notice in figure $3(\mathrm{~b})$ that the rotation becomes more important with decreasing value of $\gamma$. By observing progressive crack growth during the experiments, one can also notice that the intersection between the crack surface and a plane $(y, z)$ is always straight. This will be of importance for the geometric description of the crack surface.

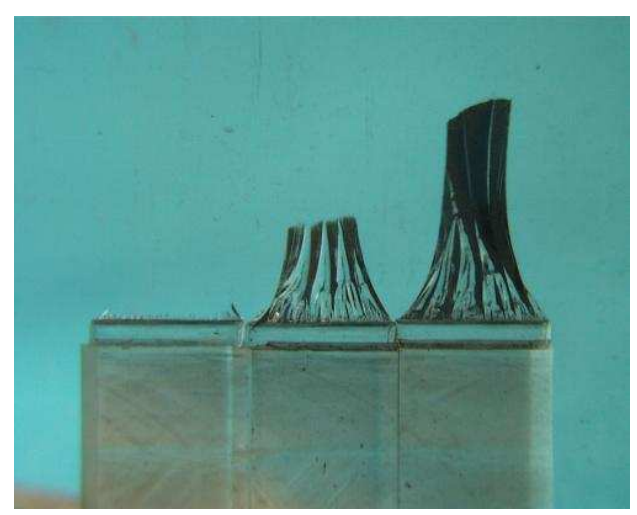

(a) Three different stages of propagation : initial facets (on the left), gradual one-piece twisting (two ones on the right) $\left(\gamma=45^{0}\right)$

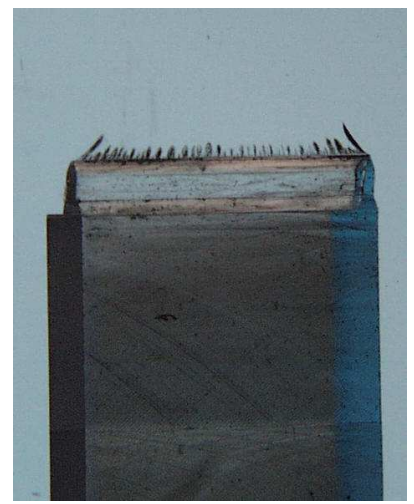

(b) Zoom on the initial facets formed by abrupt piecewise twisting

Fig. 4 Different stages of the crack propagation path

On a smaller mesoscopic length-scale, one can notice on the pictures of fig. 4 some little facets looking like abrupt factory roofs or echelon cracks, the plane of each facet being obtained by abrupt rotation of angle $\psi$ about the $x$-direction of propagation (fig. [1(d)]. Our principal aim in this paper is not to determine the angle $\psi$ locally for positions along the crack front (although it will be needed in one of the crite- 
ria) but the geometry of the macroscopic smoothly twisting crack extension.

\section{Propagation criteria}

\subsection{Preliminaries}

Figure 5(a) schematically shows the whole extension of the crack obtained experimentally, and figures 5(b) 5(c) 5(d) this same extension but restricted to a distance of propagation $\delta$ from the initial slit, $\delta$ being supposed small enough so that crack extension in each plane $x_{3}=C s t$. coincide with its tangent (fig. 5(c) $)$. Let us denote $d$ the half-length of the initial front, $\varphi\left(x_{3}\right)$ the kink angle (rotation angle of the crack extension around $O x_{3}$ ) at the point of coordinate $x_{3}$ of the front, $\varphi_{m}$ the kink angle for $x_{3}=d$. The intersection of the crack and a plane $x_{1}=\delta$ being observed to be a straight line, one easily shows (see figure 5) that $\varphi\left(x_{3}\right)$ is of the following form:

$\varphi\left(x_{3}\right)=\arctan \left(\frac{x_{3}}{d} \tan \varphi_{m}\right)$.

Since some experimenters give values of the rotation rate $\mathrm{d} \gamma / \mathrm{d} \delta$ rather than the kink angle, we give also the following relation between $\varphi_{m}$ and $\mathrm{d} \gamma / \mathrm{d} \delta$, which can easily be derived from figure 5(d)

$\frac{\mathrm{d} \gamma}{\mathrm{d} \delta}=\frac{\tan \varphi_{m}}{d}$

Nevertheless in the sequel we will focus our attention on the determination of the kink angle. The value of the rotation rate can then be deduced from equation (3).

For the determination of this kink angle, two types of criteria are proposed in the sequel. The first type predicts independently the kink angle at each point $x_{3}$ of the crack front by using the values of the SIFs at the same point $x_{3}$, hence will be called local type criteria in the sequel. These criteria are the PLS (Goldstein and Salganik, 1974), the MTS (Erdogan and Sih, 1963) and its 3D extension (Schollmann et al, 2002). The second type of criteria supposes that the kink angle is of the form of equation (2) and predicts the maximum kink angle $\varphi_{m}$ or equivalently the rotation rate $\mathrm{d} \gamma / \mathrm{d} \delta$ considering the mean value of the SIFs along the whole crack front. They will be called global type criteria in the sequel. These criteria consist in maximizing the mean value of $K_{I}$ (mode I SIF) or the mean value of $\mathcal{G}$ (total energy release rate). All these criteria are described in the following sections.

\subsection{Local type criteria}

Consider a point of the crack front and denote $K_{I}, K_{I I}, K_{I I I}$ the SIFs at this point. After propagation the crack has kinked

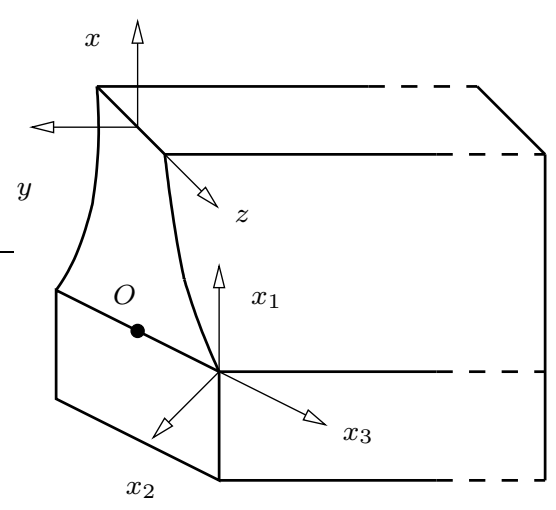

(a) Whole surface

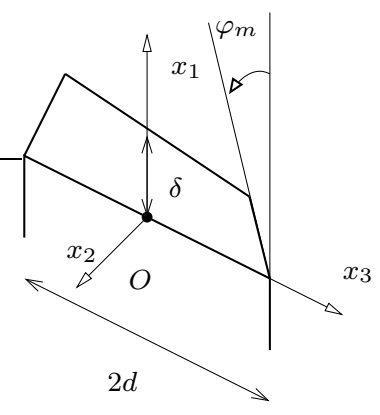

(b) $3 \mathrm{D}$ view of the local kinking

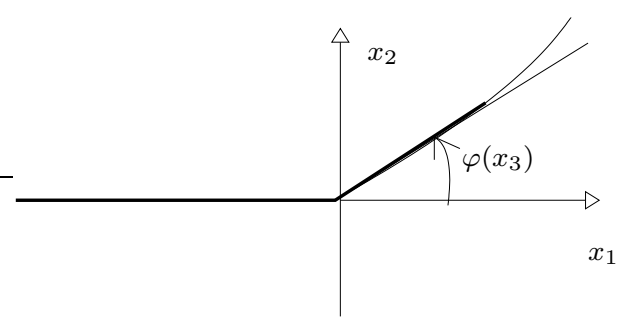

(c) In a plane $x_{3}=$ Cst.

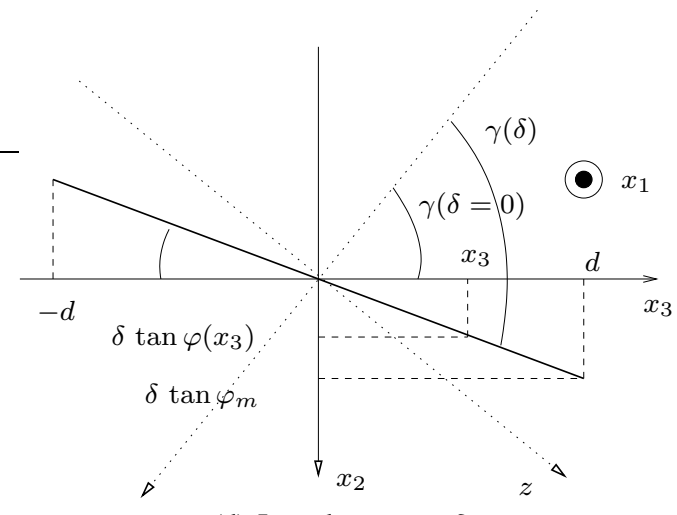

(d) In a plane $x_{1}=\delta$

Fig. 5 Schematic view of the crack surface and local kinking 
by an angle $\varphi$. We will consider the three criteria giving this kink angle versus the initial SIFs (before propagation) : the Principle of Local Symmetry (PLS), The Maximum Tangential Stress criterion (MTS), and a 3D extension of the MTS (3DMTS 1 .

The Principle of Local Symmetry states that after propagation the mode II SIF shall become zero. This yields (Amestoy an 1992):

$$
K_{I} F_{I I, I}(\varphi)+K_{I I} F_{I I, I I}(\varphi)=0
$$

where functions $F_{i, j}$ have been derived in Amestov and Leblond

1992).

The Maximum Tangential Stress criterion states that the crack propagates in the direction where the circumferential tensile stress $\sigma_{\theta \theta}$ is maximum. This yields:

$$
K_{I} \sin \varphi+K_{I I}(3 \cos \varphi-1)=0
$$

The 3D extension of the MTS developed by Schollmann et al (2002) states that the crack will grow radially from the crack front into the direction which is perpendicular to maximum principal stress $\sigma_{1}^{\prime}$. This direction corresponds to an abrupt twist angle $\psi$ of the front around the direction of propagation and a kink angle $\varphi$ which are linked to the SIFs by:

$$
\begin{aligned}
& 6 K_{I} \tan \left(\frac{\varphi}{2}\right)+K_{I I}\left(6-12 \tan ^{2}\left(\frac{\varphi}{2}\right)\right) \\
& +\left\{24\left[K_{I}-3 K_{I I} \tan \left(\frac{\varphi}{2}\right)\right]\right. \\
& \cdot\left[K_{I} \tan \left(\frac{\varphi}{2}\right)+K_{I I}\left(1-2 \tan ^{2}\left(\frac{\varphi}{2}\right)\right)\right] \\
& \left.+32 K_{I I I}^{2} \tan \left(\frac{\varphi}{2}\right) \cdot\left(1+\tan ^{2}\left(\frac{\varphi}{2}\right)\right)^{2}\right\} \\
& \cdot\left\{\left[4 K_{I}-12 K_{I I} \tan \left(\frac{\varphi}{2}\right)\right]^{2}\right. \\
& \left.+64 K_{I I I}^{2}\left(1+\tan ^{2}\left(\frac{\varphi}{2}\right)\right)^{2}\right\}^{-1 / 2}=0
\end{aligned}
$$

Since PLS and MTS criterion give nearly the same value of the kink angle 2 , we will only consider in the sequel the MTS and 3DMTS criterion.

\subsection{Global type criterion of Lazarus and Leblond}

Starting from the idea that the twisting of the crack front is due to the presence of mode III, a criterion linked to the disappearance of mode III has to be derived. This criterion is necessary of global type (that is involving all the points of

\footnotetext{
${ }^{1}$ Other criteria exist, see Oian and Fatemi (1996), but the aim here is not to make a systematic review of these local criteria but to compare two types (local and global) of criteria.

2 Indeed Amestov and Leblond (1992) have shown that the difference is of order $m^{6}$ with $m=\varphi / \pi$
}

the front) since this twisting around the direction of propagation can only be achieved if all the points twists together. Lazarus et al. (1998; 2001b) proposed two such criteria:

MVG: maximize the Mean Value of $\mathcal{G}\left(x_{3}, \delta\right)$ along the front with respect to $\varphi_{m}$

Mrin IIOnlt with respect to $\varphi_{m}$

where $\mathcal{G}\left(x_{3}, \delta\right)$ denotes the total energy release rate and $K_{I}\left(x_{3}, \delta\right)$ the mode I SIF, both after a propagation over a distance $\delta$ ind at the point of coordinate $x_{3}$ of the front. One has:

$$
\mathcal{G}\left(x_{3}, \delta\right)=\frac{1-\nu^{2}}{E}\left[K_{I}^{2}+K_{I I}^{2}\right]\left(x_{3}, \delta\right)+\frac{1+\nu}{E} K_{I I I}^{2}\left(x_{3}, \delta\right)
$$

Applying these criteria for a vanishing $\delta$, that is, a vanishing crack extension length, one gets a zero value for $\varphi_{m}$.

This implies that the criteria must be applied after a nonzero propagation length. On figure 4(b) one observes the presence of little facets at the beginning of the propagation. During the formation of the facets, the points of the crack front propagate in an "individual" manner. Afterwards, as soon as the facets have disappeared and the entire front twists as a whole, their behaviour becomes "collective". From there derives the idea

to fix $\delta$ in the criteria to the experimentally measured length
\[ \delta_{c} \text { of these little facets. } \]

In order to apply the criteria, let us suppose the length $\delta_{c}$ of the extension to be small enough for the expansion of the stress intensity factors $K_{I}\left(x_{3}, \delta\right), K_{I I}\left(x_{3}, \delta\right), K_{I I I}\left(x_{3}, \delta\right)$ in powers of the crack extension length $\delta$ obtained by Leblond (1989) and Lazarus et al (2001a) to be applicable. These expressions give the SIFs after propagation $K_{p}\left(x_{3}, \delta\right)$ in function of the initial SIFs $K_{p}\left(x_{3}\right), p=I, I I, I I I$. Denote $k_{p}$ the mean value of these quantities:

$k_{p}=\frac{1}{2 d} \int_{-d}^{d} K_{p}\left(x_{3}\right) d x_{3}$

If $\left|k_{I I I} / k_{I}\right| \ll 1$ and $\left|k_{I I} / k_{I}\right| \ll 1$, the criteria MVK and MVG then give respectively the following formulas for $\varphi_{m}$ :

$$
\varphi_{m}=\frac{8}{1+\frac{16 \sqrt{2}}{3 \pi} \sqrt{\frac{\delta_{c}}{a}}-\frac{5}{12} \frac{\delta_{c}}{a}+\frac{6}{\pi} \frac{2-3 \nu}{2-\nu} \frac{\delta_{c}}{d}} \frac{\delta_{c}}{d} \frac{k_{I I I}}{k_{I}}
$$

$$
\varphi_{m}=\frac{4}{(2-\nu)\left(\frac{1}{3}+\frac{4 \sqrt{2}}{3 \pi} \sqrt{\frac{\delta_{c}}{a}}-\frac{16}{3 \pi^{2}} \frac{\delta_{c}}{a}+\frac{2}{\pi} \frac{2-3 \nu}{2-\nu} \frac{\delta_{c}}{d}\right)} \frac{\delta_{c}}{d} \frac{k_{I I I}}{k_{I}}
$$


(10)

These theoretical expressions of $\varphi_{m}$ were obtained analytically for $\left|k_{I I I} / k_{I}\right| \ll 1$ and $\left|k_{I I} / k_{I}\right| \ll 1$. However, $\varphi_{m}$ can be computed numerically for arbitrary values of $K_{I I I}\left(x_{3}\right) / K_{I}\left(x_{3}\right)$ and $K_{I I}\left(x_{3}\right) / K_{I}\left(x_{3}\right)$. The results show that the analytic formulae are valid even for $k_{I I I} / k_{I}$ and $k_{I I} / k_{I}$ of order unity.

It is logical to assume that the initial facets, which are deduced from the initial slit by a rotation of an angle $\psi$ about the $O x_{1}$ axis, disappear when they merge into the global crack surface which gradually rotates about the same axis with the rotation rate $\frac{\mathrm{d} \gamma}{\mathrm{d} \delta}$. Hence, the length $\delta_{c}$ is linked to the rotation rate by the following formula:

$\frac{\mathrm{d} \gamma}{\mathrm{d} \delta} \delta_{c}=\psi$

In this expression, $\psi$ may be evaluated through Eq. (1) and $\frac{\mathrm{d} \gamma}{\mathrm{d} \delta}$ through Eqs. (3) and, (9) or (10). Since $\varphi_{m}$ itself depends on $\delta_{c}$, Eq. 11 in fact yields an equation on this quantity which must be solved numerically to obtain $\delta_{c}$ as a function of $K_{I I I} / K_{I}$. Associated with Eqs. (9) or (10) the criterion gives finally $\varphi_{m}$ as a function of $K_{I I I} / K_{I}, a, d$, and $\nu$.

\section{Comparison with the experiments}

\subsection{Determination of the SIF}

The Stress Intensity Factors are computed using the program ADAPCRACK3D developed at the Institute of Applied Mechanics at University of Paderborn (Fulland et al, 2000; Schollmann et al, 2003). It is a Finite-Element based tool for simulation of crack propagation processes in threedimensional structures. The program delegates the determination of the mechanical fields to the commercial FE-Solver ABAQUS. Using these fields, the SIFs for all three fracture modes are then determined by the module NETCRACK3D by the use of the MVCCI-method Rvbicki and Kanninen, 1977; Buchholz, 1984). They are given in figure 6 for one half of the front (the other part can be deduced from the fact that due to symmetry considerations, $K_{I I} / K_{I}$ is an odd function of $x_{3}$ and $K_{I I I} / K_{I}$ is an even one). Results for the points located at points $x_{3}= \pm d$ shall be disregarded since the classical SIFs cannot be defined at these free surface points (Bazant and Estenssoro, 1979) due to the corner singularity, which differs from $-1 / 2$. One observes that $K_{I I I}$ predominates over $K_{I I}$, that $K_{I I I} / K_{I}$ is nearly uniform and increases while $\gamma$ decreases (more inclined notch).

\subsection{Comparison between MTS, 3D-MTS, MVK and MVG}

Figures 7 and 8 compare the experimental kink angle along the front with the ones obtained by, on the one hand, MTS

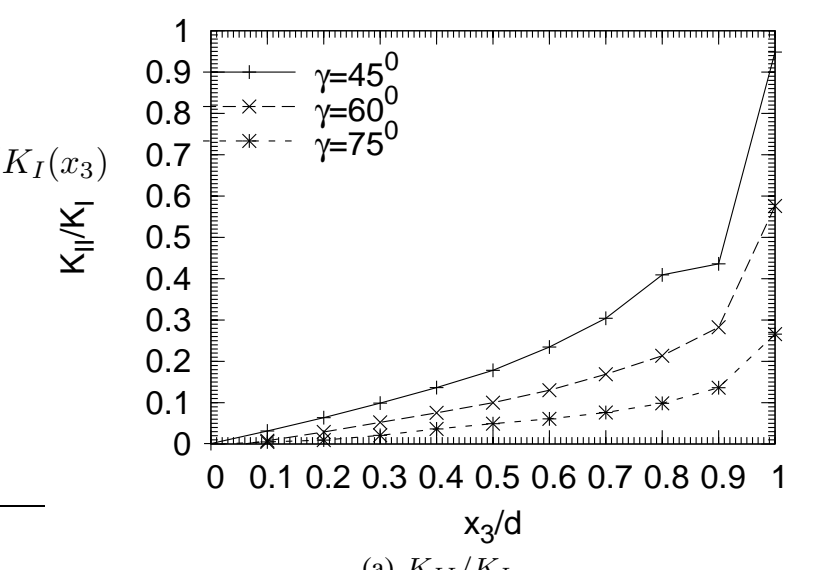

(a) $K_{I I} / K_{I}$

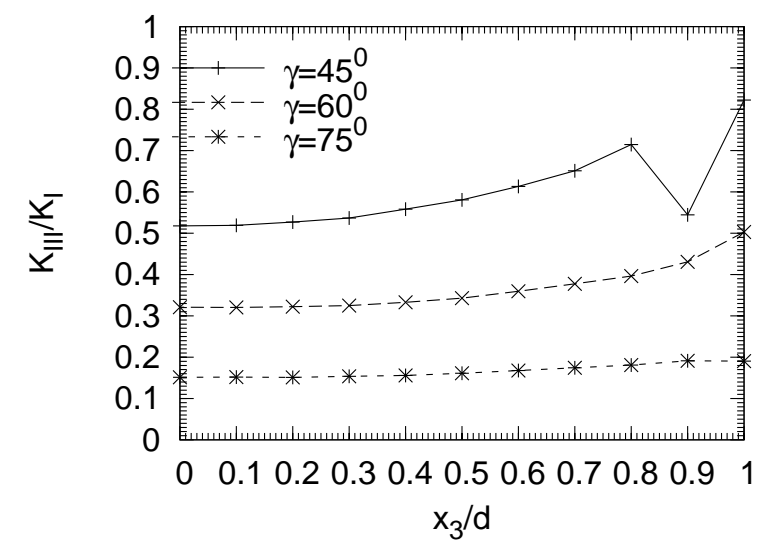

(b) $K_{I I I} / K_{I}$

Fig. 6 Stress Intensity Factors for the initial slit

and 3DMTS criteria and on the other hand, MVK and MVG criteria (again only the values for one half of the front is plotted thanks to symmetry and external points shall be disregarded since SIF are not defined at these points). Experimental kink angles are obtained by measuring the kink angle $\varphi_{m}$ at the free surfaces $x_{3}= \pm d$ and using equation (2) to obtain them for the interior points of the front.

The following observations become apparent:

1. Despite the two concepts rely on totally different aspects of the loading situation of the crack front both predictions of the kinking angle are quite satisfactory.

2. For the MTS and 3DMTS criteria, an excellent agreement is found near the free surfaces. But the kink angles are notably underestimated in the middle of the front. This is not surprising, since the small initial facets, which could be observed in the experiments, suggest that the twisting linked to mode III, is of relevance in those cases. Due to its omission, the twisting of the whole crack front is underestimated.

3. For the MVK and MVG criteria an excellent agreement with the experiments can be found. The relative errors 


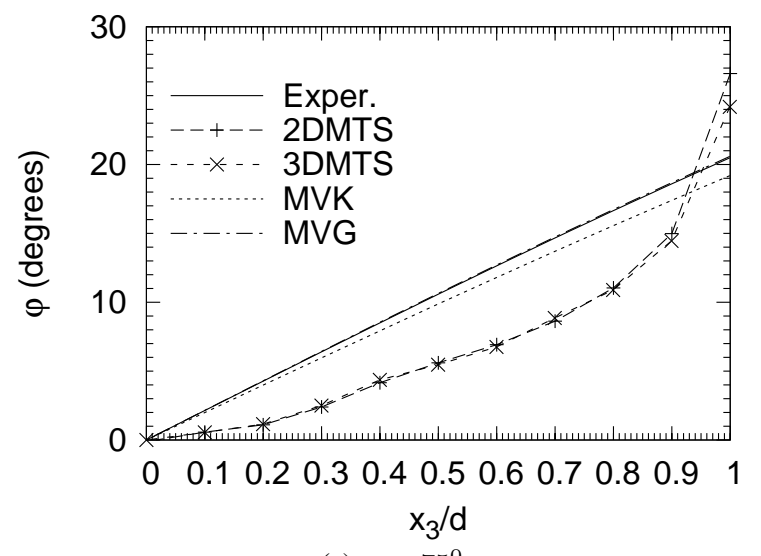

(a) $\gamma=75^{\circ}$

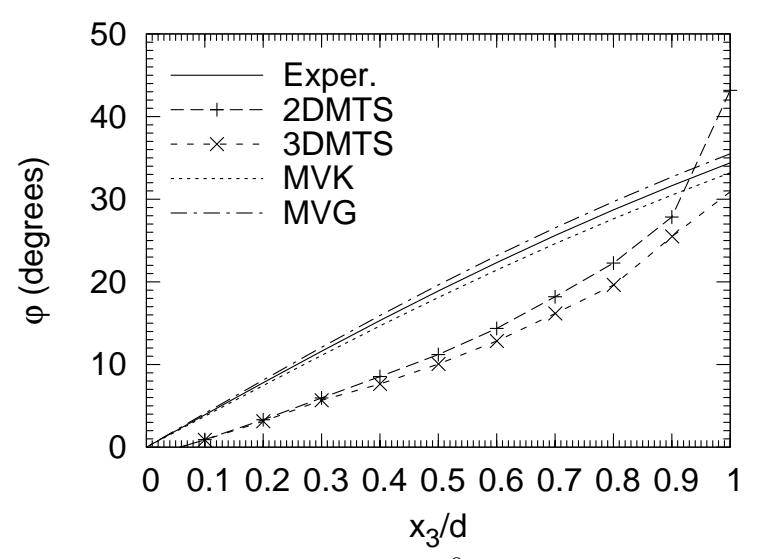

(b) $\gamma=60^{\circ}$

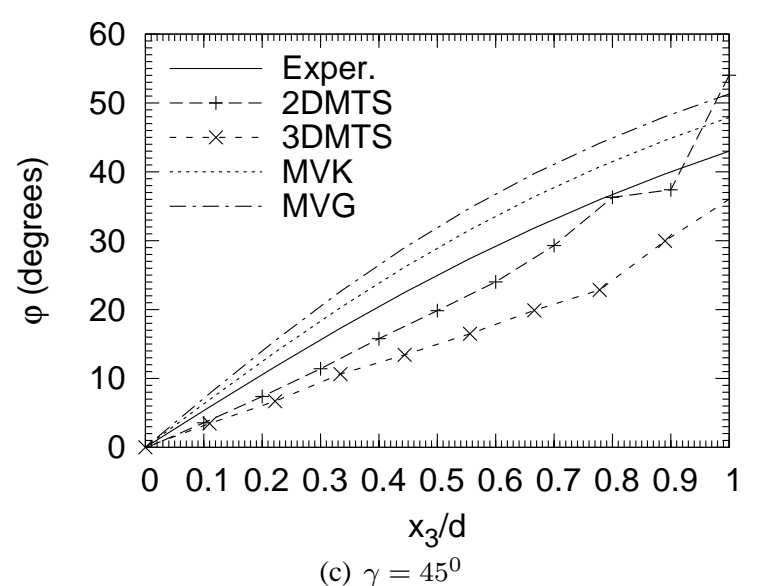

Fig. 7 Comparison of MTS, 3DMTS and MVK, MVG

are less than $5 \%$ for $\gamma=75^{0}, 60^{\circ}$ and about $20 \%$ for $\gamma=45^{0}$. The error increase is probably due to the lack of taking $K_{I I}$ into account and to the use of a first order expression of $\varphi_{m}$ with respect to $K_{I I I} / K_{I}$.

4. For $\gamma=75^{0}, 60^{0}$ MTS and 3DMTS give very similar results, but for $\gamma=45^{0}$, MTS seems to be more ac-

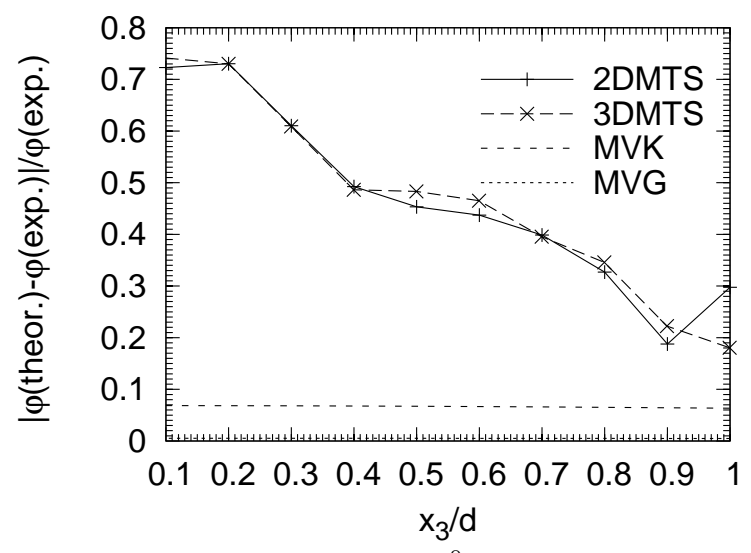

(a) $\gamma=75^{\circ}$

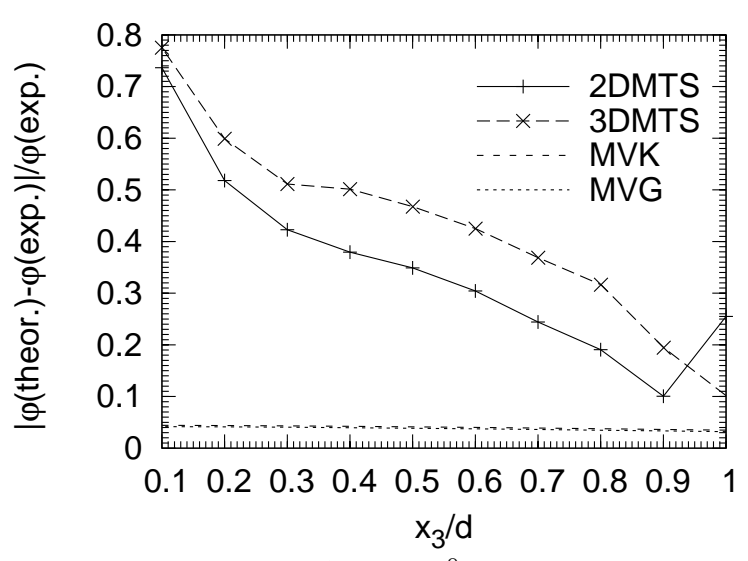

(b) $\gamma=60^{0}$

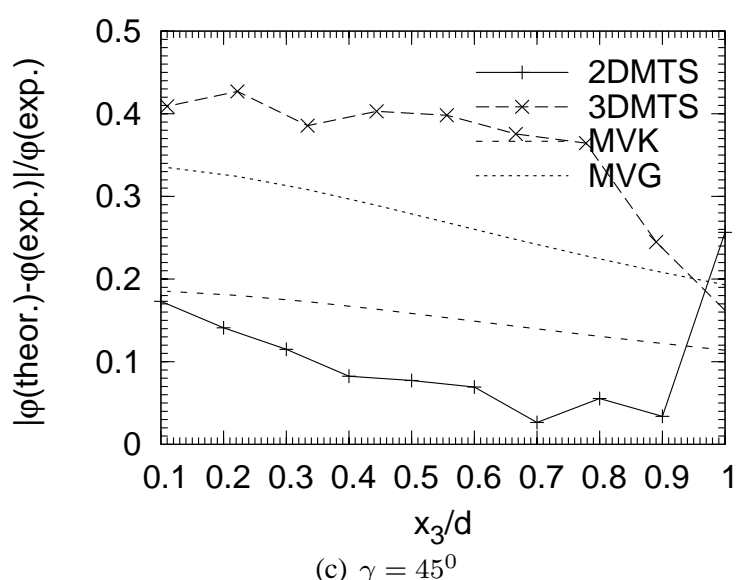

Fig. 8 Relative error

curate. It seems that the kink angle is better predicted by maximize the opening stress on facets containing the crack front (MTS) than on any facets in front of the tip (3DMTS). Hence even in presence of mode III, MTS or equivalently PSL shall be used rather than (3DMTS). 
5. Criteria MVK and MVG yield very similar values, hence are difficult to discriminate by the experiments. Nevertheless, a more precise estimation of the errors gives a slight advantage to MVK. It was still the case in Lazarus et al (2001b) for similar experiments made on steel.

\subsection{Propagation path by ADAPCRACK3D}

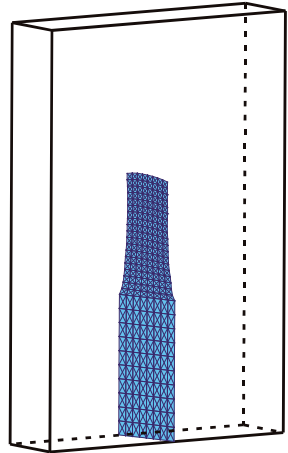

(a) $\gamma=75^{0}$

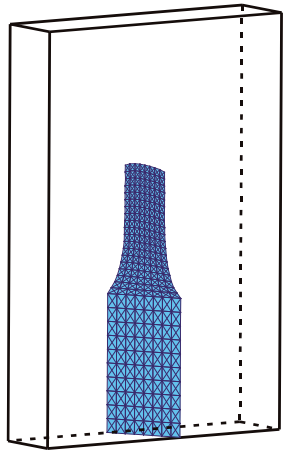

(b) $\gamma=60^{\circ}$

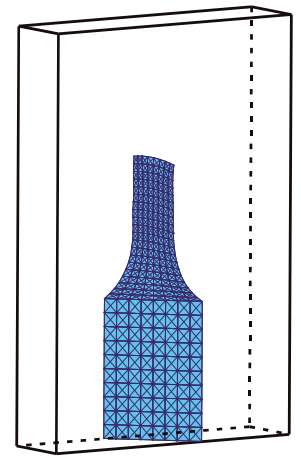

(c) $\gamma=45^{0}$

Fig. 9 Computed crack paths

Even if global type criteria give better prediction for the initial kink angle, application of the local type ones iteratively during propagation gives a crack surface very similar to the experiments (see fig. 9 compared to 3(a). This demonstrates the ability of ADAPCRACK3D to perform simulations of $3 \mathrm{D}$ crack propagation paths, even if the initial kink angle is slightly underestimated. The mesh and the deformation of the specimen during computation is depicted in figure 10

\section{Conclusion}

By comparison of the criteria with the experiments, one can notice that:

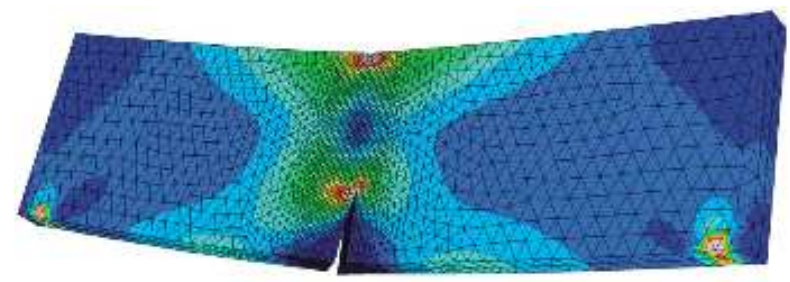

(a) With initial crack

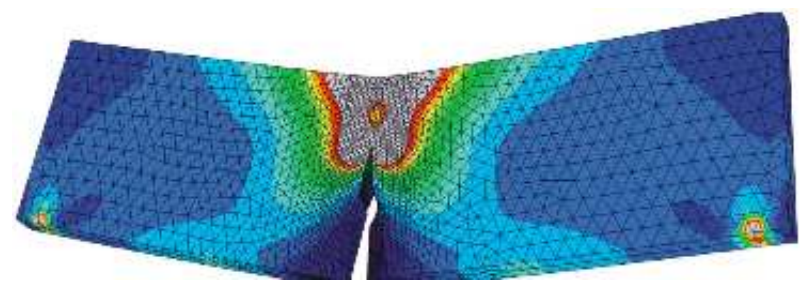

(b) After 20 steps of simulated incremental fatigue crack growth

Fig. 10 Deformed FE-model of the 3PB-specimens $\left(\gamma=45^{0}\right.$, displacement magnification factor $\mathrm{DMF}=50$ )

- The presence of both mode II and mode III along the crack front implies that both investigated criteria give good results concerning the development of the crack front in 3PB specimen under notable mode III influence.

- The global type criteria by Lazarus and Leblond, which was especially designed for those crack cases, predict the macroscopic deflection of the crack front by supposing that the whole front twists around the direction of propagation to annihilate mode III. Its results match the experimentally obtained crack development with excellent accuracy. But the criterion, specially designed for these experiments, seems difficult to be extended to any other configuration.

- The local type MTS criteria predict crack growth by definition of a local kinking angle that annihilate mode II. They differ more from the experimental findings than the predictions of the criterion by Lazarus and Leblond, since they disregard the local twisting $\psi$ due to mode III. But they are of greater universality, as it is applicable to arbitrary 3D-crack problems.

- Moreover from a global point of view the smaller rotation rate resulting from the underestimation of the crack front twisting due to the disregard of the twisting angle does not notably change the overall shape of the final crack surface. Hence MTS criterion seems sufficient for any practical engineering purposes.

It can finally be concluded, that obviously the crack front rotation, which is observed under notable mode III influence in 3PB specimens, can be described by both local and global type criteria. Hence, it is not possible to definitely conclude whether the twisting observed in $3 \mathrm{~PB}$ or $4 \mathrm{~PB}$ is a mode II or a mode III effect. But since the global type criterion gives 
better results, one shall conclude that it is rather a mode III one.

Now, a more extensive experimental study of crack propagation paths in presence of mode III shall be done. The conditions of transition between these several crack patterns (abrupt or progressive, one-piece or piecewise twisting, see fig. 1) shall be lightened. The size effects, loading conditions on the spacing and rotation angle of the factory-roof patterns shall be studied. Extensive comparison of the twisting angle $\psi$ of 3DMTS criterion or the empiric formula (11) with the twisting of the facets are envisaged in a near future.

\section{References}

Amestoy M, Leblond JB (1992) Crack Paths in Plane Situations - II. Detailed Form of the Expansion of the Stress Intensity Factors. International Journal of Solids and Structures 29:465-501

Aydin A, Pollard DD (1988) Progress in understanding jointing over the past century. Geological Society of America Bulletin 100:1181-1204

Bazant Z, Estenssoro L (1979) Surface singularity and crack propagation. International Journal of Solids and Structures 15(5):405-26

Buchholz FG (1984) Improved formulae for the finite element calculation of the strain energy release rate by the modified crack closure integral method. In: Robinson J (ed) Accuracy, Reliability and Training in FEM Technology, Robinson and Associates, Dorset, pp 650-659

Buchholz FG, Wang H, Lin J, Richard HA (1998) 3d fracture analyses and experimental results on three-point bend specimens with slant cracks. In: Atluri SN, O’Donoghue PE (eds) Modeling and Simulation based engineering. Proceedings of the International Conference of Engineering science (ICES 98), Tech Science Press, Palmdale, Atlanta, Georgia, USA, vol II, pp 1238-1243

Buchholz FG, Chergui A, Richard HA (2004) Fracture analyses and experimental results of crack growth under general mixed mode loading conditions. Engineering Fracture Mechanics 71(4-6):455-468

Buchholz FG, Wiebesiek J, Fulland M, Richard H (2005) Comparison of computational $3 \mathrm{~d}$ crack path simulations with experimental findings for 3pb-specimens with inclined crack planes. In: M H Aliabadi JAe F-G Buchholz (ed) Advances in Fracture and Damage Mechanics IV. Proceedings of the 4th International Conference on Fracture and Damage Mechanics (FDM05), EC Ltd., UK, Mallorca, Spain, pp 143-148

Cooke ML, Pollard DD (1996) Fracture propagation paths under mixed mode loading within rectangular blocks of polymethyl methacrylate. Journal of Geophysical Research 101(B2):3387-3400
Erdogan G, Sih GC (1963) On the crack extension in plates under plane loading and transverse shear. ASME J Basic Engng 85:519-527

Fulland M, Schöllmann M, Richard HA (2000) Adapcrack3d-development of the program for the simulation of three-dimensional crack propagation processes. In: Atluri SN, Brust FW (eds) Advances in Comp. Engineering \& sciences, Tech Science Press, Palmdale, USA, pp 948-953

Goldstein RV, Salganik RL (1974) Brittle fracture of solids with arbitrary cracks. International Journal of Fracture 10:507-523

Gravouil A, Moes N, Belytschko T (2002) Non-planar 3d crack growth by the extended finite element and level sets. II. Level set update. International Journal for Numerical Methods in Engineering 53(11):2569-86

Hourlier F, Pineau A (1979) Fissuration par fatigue sous sollicitations polymodales (mode I ondulé + mode III permanent) d'un acier pour rotors 26 NCDV 14. Mémoires Scientifiques de la Revue de Métallurgie pp 175-185

Hull D (1993) Tilting cracks: the evolution of fracture surface topology in brittle solids. International Journal of Fracture 62(2):119-138

Hull D (1999) Fractography. Press syndicate of the university of Cambridge, UK

Lazarus V, Leblond JB (1998) Crack paths under mixed mode $(\mathrm{I}+\mathrm{III})$ or $(\mathrm{I}+\mathrm{II}+\mathrm{III})$ loadings. Comptes Rendus de l'Académie des Sciences, Série II (Mécanique, Physique, Astronomie) 326(3):171-177

Lazarus V, Leblond JB, Mouchrif SE (2001a) Crack front rotation and segmentation in mixed mode I+III or I+II+III - part I: Calculation of Stress Intensity Factor. Journal of the Mechanics and Physics of Solids 49(7):1399-1420

Lazarus V, Leblond JB, Mouchrif SE (2001b) Crack front rotation and segmentation in mixed mode I+III or I+II+III - part II: Comparison with experiments. Journal of the Mechanics and Physics of Solids 49(7):1421-1443

Leblond JB (1989) Crack Paths in Plane Situations - I. General Form of the Expansion of the Stress Intensity Factors. International Journal of Solids and Structures 25:13111325

Makabe C, Anggit M, Sueyoshi T, Yafuso T (2006) The formation mechanism of the factory-roof pattern in a torsional fatigue specimen with circumferential notch. J Testing Evaluation 34(5):423-429

Palaniswamy K, Knauss WG (1975) Crack extension in brittle solids. Mechanics Today, Pergamon Press 4:87

Pollard DD, Segall P, Delaney PT (1982) Formation and interpretation of dilatant echelon cracks. Geological Society of America Bulletin 93:1291-1303

Pook L (1995) On fatigue crack paths. International Journal of Fatigue 17(1):5-13 
Qian J, Fatemi A (1996) Mixed mode fatigue crack growth: a literature survey. Engineering Fracture Mechanics 55(6):969

Rybicki EF, Kanninen MF (1977) A finite element calculation of stress intensity factors by a modified crack closure integral. Engineering Fracture Mechanics 9:931-938

Schollmann M, Richard H, Kullmer G, Fulland M (2002) A new criterion for the prediction of crack development in multiaxially loaded structures. International Journal of Fracture 117(2):129-41

Schollmann M, Fulland M, Richard HA (2003) Development of a new software for adaptive crack growth simulations in $3 d$ structures. Engineering Fracture Mechanics 70(2):249- 268

Sommer E (1969) Formation of fracture 'lances' in glass. Engineering Fracture Mechanics 1:539-546

Yates JR, Mohammed RA (1996) Crack propagation under mixed mode (I+III) loading. Fatigue \& Fracture Engineering Materials \& Structures 19(10):1285-1290 